\title{
Effects of Electrical Stimulation at Different Locations in the Central Nucleus of Amygdala on Gastric Motility and Spike Activity
}

\author{
Feng $\mathbf{H E}^{1}$, Hong-Bin $\mathbf{A} \mathbf{I}^{1}$ \\ ${ }^{1}$ Key Laboratory of Animal Resistance of Shandong Province, College of Life Sciences, Shandong \\ Normal University, Jinan, Shandong Province, People's Republic of China
}

Received July 9, 2015

Accepted September 18, 2015

On-line March 15, 2016

\begin{abstract}
Summary
The aim of the study was to determine the effects of electrical stimulation of different locations in the central nucleus of amygdala (CNA) on gastric motility and spike activity in dorsal vagal complex. Gastric motility index (GMI) and firing rate (FR) of dorsal vagal complex neurons were measured in adult Wistar rats respectively. Neuronal spikes in dorsal vagal complex (DVC) were recorded extracellularly with single-barrel glass microelectrodes. Each type of responses elicited by electrical stimulation in medial (CEM) and lateral (CEL) subdivisions of CNA were recorded, respectively. GMI was significantly increased after stimulation of CEM $(p<0.01)$, and significantly decreased in response to CEL stimulation $(p<0.01)$. After stimulation of CEM, FR in medial nucleus of the solitary tract (mNST) decreased by $31.6 \%$ $(p<0.01)$ and that in dorsal motor nucleus of the vagus (DMNV) increased by $27.1 \%(p<0.01)$. On the contrary, FR in mNST increased $(p<0.01)$ and that in DMNV decreased in response to CEL stimulation $(p<0.05)$. In conclusions, our findings indicated that different loci of CNA may mediate differential effects on gastric activity via changes in the firing of brainstem neurons controlling gut activity.
\end{abstract}

\section{Key words}

Central nucleus of the amygdala • Gastric motility • Neuronal spikes - Medial nucleus of the solitary tract - Dorsal motor nucleus of the vagus

\section{Corresponding author}

H.-B. Ai, Key Laboratory of Animal Resistance of Shandong Province, College of Life Sciences, Shandong Normal University, No.88, East Wenhua Road, Lixia District, Jinan, P. R. China 250014. E-mail: hongbinaiaiai@163.com

\section{Introduction}

Gastric motility is a hot topic in the motor physiology research of the stomach in health and disease (Cullen and Kelly 1993, Kim et al. 2014). Both increases or increases in gastric motility can induce different gastric dysfunctions, and for example stress-induced gastric lesions may be caused by the alterations in motility pattern (Grandi et al. 2007). Inhibition of gastric motility induces the delay of gastric emptying, which is a common symptom of functional dyspepsia and irritable bowel syndrome (Stanghellini et al. 2002, Talley et al. 2006). Alterations in gastric motility and gastrointestinal disorders are often associated with responses to certain types of emotion, such as fear and anxiety (Huerta-Franco et al. 2012, Porcelli et al. 2014, Zádori and Gyires 2013).

The central nucleus of the amygdala (CNA) has an important role in response to emotion (Grèzes et al. 2014, Kim et al. 2011), such as fear and anxiety (Duvarci et al. 2011, Pare and Duvarci 2012, Ventura-Silva et al. 2013, Zádori and Gyires 2013). Many anatomical studies have demonstrated that CNA is connected to the dorsal vagal complex (DVC), the primary center for controlling gastrointestinal functions (Awan and Rutherford 2011, Hornby and Wade 2011, Zhang et al. 2003). And some physiological studies have shown that stimulation of CNA can evoke the change in gastric motility via DVC (Liubashina et al. 2000, Rinaman and Koehnle 2010, Zhang et al. 2003).

CNA can be further divided into lateral (CEL) and medial (CEM) regions that have different functions (Ciocchi et al. 2010). Previous studies have reported that electrical stimulation of different regions of amygdala 
(CEL and CEM) can induce diverse vagal-dependent effects on gastric motor activity, indicating that CEL and CEM have varied functions in the mediation of gastrointestinal activities (Lyubashina 2004). Furthermore, efferent fibers from CNA terminate in the nucleus of the solitary tract (NST) and dorsal motor nucleus of the vagus (DMNV) in gastrointestinal-associated regions (Zhang et al. 2003). Whether CNA modulates gastrointestinal activities via NST and DMNV remains unknown.

In the present study, through electrical stimulation of CEM and CEL respectively, we attempted to investigate the roles of different CNA regions, as well as NST and DMNV in modulating gastric motility by measuring gastric motility index, as well as neuronal discharge rates in the medial NST (mNST) and DMNV. Interestingly, the results obtained here are opposite to those reported by Lyubashina (2004) previously. The results are relevant to the mechanisms mediating emotional influences on gastric motility, and suggest possible complexity in the factors that determine specific patterns of physiological response to amygdalar regional activation.

\section{Materials and Methods}

\section{Animal preparation}

All experiments were performed on adult Wistar rats (250-300 $\mathrm{g}$ of weight) purchased from the Experiment Animal Center of Shandong University, China. Rats were kept in a temperature-controlled room $\left(22 \pm 2{ }^{\circ} \mathrm{C}\right)$ under normal day/night cycle with no restriction to food and water. All experimental procedures were approved by the Department of Medical Ethics School of Medicine Shandong University and conducted in accordance with the Guide for the Care and Use of Laboratory Animals (Institute for Laboratory Animal Research, National Research Council 1996).

\section{Electrical stimulation of different subdivisions of CNA}

Rats were carefully placed in a prone position and were fixed with a double-arm animal stereotaxic frame (68002, RWD Life Science, China). Limited craniotomy was performed according to the position where stimulating or recording electrode was to be planted. Electrical stimulation of CNA was performed with lacquer-insulated monopolar, stainless-steel electrodes (tip diameter of $50 \mu \mathrm{m}$, resistance of 15 $20 \mathrm{k} \Omega$ ). Based on the stereotaxic coordinates of rat brain (Paxinos and Watson 1998), the tip of electrode were positioned at the following coordinates: CEM (P: 1.8$2.4 \mathrm{~mm}$ posterior to bregma; $\mathrm{L}: 3.5-4.0 \mathrm{~mm}$ lateral to the midline; H: $8.0-8.5 \mathrm{~mm}$ ventral to the brain skull surface) (Fig. $1 \mathrm{~A}$ ) and CEL (P: 2.0-2.8 mm; L: 4.3-4.8 mm; H: 7.8-8.2 mm) (Fig. $1 \mathrm{~B}$ ), respectively. Single squarewave pulses (duration of $0.5 \mathrm{~ms}$, amplitude of $0.2 \mathrm{~mA}$ ) were delivered at a frequency of $30 \mathrm{~Hz}$ for $30 \mathrm{~s}$ by a Programmable Stimulator (Y2, Chengdu Instrument Factory, China). Changes in gastric motility were recorded at $3 \mathrm{~min}$ before and $3 \mathrm{~min}$ after electrical stimulation respectively.
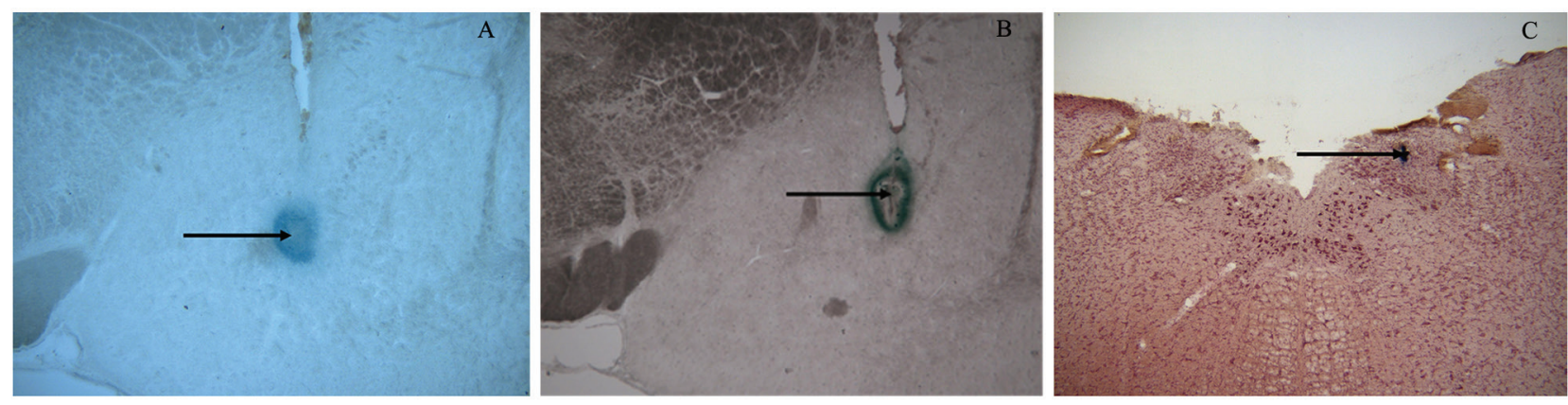

Fig. 1. Visualization of electrical stimulation positions using Pontamine sky blue or together with neutral red staining. A. Electrical stimulation position in the lateral part (CEL) of the central nucleus of amygdale; B. Electrical stimulation position of the medial (CEM) part of the central nucleus of amygdala regions; C. Electrical stimulation position visualized by Pontamine sky blue in a neutral redstained section.

\section{Determination of gastric motility}

Gastric motility was determined by the rubberballoon method (Zádori and Gyires 2013), a widely used method for the measurement of gastric motility (Zádori and Gyires 2013). Briefly, after fasted for $24 \mathrm{~h}$, rats were anesthetized with $4 \%$ chloral hydrate (400 mg/kg i.p.). Rats were kept in a thermostatically controlled heating blanket $\left(37 \pm 1^{\circ} \mathrm{C}\right)$ during the progressing of all the 
experimental procedures. To record the changes in gastric motility, a midline laparotomy was performed. A latex balloon attached to a thin polyethylene tube was leaned into the stomach via fundus, and positioned at corpus/antrum area. Then the balloon was inflated with $2 \mathrm{ml}$ of warm distilled water to produce global distention of the stomach and to achieve a baseline intragastric pressure (8-12 $\mathrm{mm} \mathrm{Hg}$ ). The distal end of tubing was connected to a pressure transducer (Chengdu Taimeng, China) and to a BL-420 Biological Experimental System (Chengdu Taimeng, China) to monitor intragastric pressure.

\section{Neuronal spikes in the DVC}

Electrical stimulation was performed as described above. Neuronal spikes were recorded extracellularly with single-barrel glass microelectrodes (tip diameters of 1-2 $\mu \mathrm{m}$; resistance of 8-15 M $\Omega$ ), which were filled with $0.5 \mathrm{M}$ sodium acetate and $2 \%$ Pontamine sky blue. The glass microelectrode was lowered slowly into DMNV and mNST, and the stereotaxic coordinates were as follows: DMNV (A: 0.5-1.0 mm anterior to obex; $\mathrm{L}$ : $0.4-0.6 \mathrm{~mm}$ lateral to the midline; $\mathrm{H}: 0.5-0.7 \mathrm{~mm}$ ventral to dura) and $\operatorname{mNST}(\mathrm{A}: 0.5-1.0 \mathrm{~mm}$; $\mathrm{L}$ : 0.3-0.5 mm; H: 0.2-0.4 mm) (Fig. $1 \mathrm{C}$ ). The brain was covered with $3 \%$ agar in saline in order to reduce the influence of ventilation and heartbeat. Potential was amplified using a microelectrode bridge amplifier (ME200A, Chengdu Taimeng, China) and continuously recorded with bandpass-filler (160-1000 Hz) by BL-420 Biological Experimental System. All data stored on disk were used for off-line analysis.

\section{Histological identification}

At the end of the experiments, histological verification was done to check the position of stimulating and recording electrodes. Cathodal direct current $(-0.1 \mathrm{~mA}, 10 \mathrm{~s})$ was passed through stimulating electrode to form $\mathrm{Fe}^{3+}$ deposit into the stimulating site in the CEA. Anodic direct current $(0.01 \mathrm{~mA}, 20 \mathrm{~min})$ was passed through recording electrode to form an iron deposit of Pontamine sky blue into the recording site. Then, all the rats were deeply anesthetized with an overdose urethane and perfused transcardially with $0.9 \%$ sodium chloride solution followed by $1 \%$ potassium ferrocyanide and $10 \%$ formalin solution. The potassium ferrocyanide was used to react with $\mathrm{Fe}^{3+}$ and produced Prussian blue which can be identified clearly. After decapitation brains were removed and post-fixed in a mixture of $10 \%$ formalin and $20 \%$ sucrose solution for at least $24 \mathrm{~h}$. Then the brains were cut into $40-\mu \mathrm{m}$ thick coronal serial sections. The locations of stimulating and recording sites were determined microscopically, with neutral red staining if necessary. Only data collected from correct positions (as shown in Fig. 1) were used for later statistical analysis.

\section{Data analysis}

Gastric motility index (GMI), defined as the sum of amplitude and duration of all gastric contraction waves in a unit time, was used to quantify gastric motility. GMI was quantified manually and calculated following the formula:

$\mathrm{GMI}=\left(\mathrm{T}_{1} \times \mathrm{A}_{1}+\mathrm{T}_{2} \times \mathrm{A}_{2}+\ldots+\mathrm{T}_{\mathrm{n}} \times \mathrm{A}_{\mathrm{n}}\right) /\left(\mathrm{T}_{1}+\mathrm{T}_{2}+\ldots+\mathrm{T}_{\mathrm{n}}\right)$

" $\mathrm{T}$ " represents the duration of gastric contraction wave in a unit time (s) and " $\mathrm{A}$ " represents the amplitude of gastric contraction wave ( $\mathrm{mm} \mathrm{Hg}$ ). Firing rate (FR, spikes/s) was used to quantify neuronal activity in the target nucleus.

All the data were denoted as mean \pm standard error (SE). GMI and FR at $3 \mathrm{~min}$ before and $3 \mathrm{~min}$ after electrical stimulation were compared by paired-samples $t$ test under each treatment respectively. Independentsample $t$ tests were used, if necessary, to compare between CEM and CEL groups. All statistical analysis was performed by SPSS16.0 software (SPSS Inc. Chicago, IL., USA) and $p<0.05$ was chosen as the cut-off criterion.

\section{Results}

Effects of electrical stimulation of CEM and CEL on gastric motility

Prior to electrical stimulation, GMI of CEM $(n=10)$ and CEL $(n=10)$ groups were 1008.4 \pm 109.1 and $995.3 \pm 77.7$ respectively, with no significant difference ( $p>0.05$, Fig. 2 C). Electrical stimulation of CEM led to sharp increase in intragastric pressure (IGP) (Fig. 2 A) and evoked significant increase in GMI $(p<0.01)$ from $1008.4 \pm 109.1$ to $1499.7 \pm 155.4$ (Fig. 2 C). By contrast, significant decreases in IGP and GMI (from 995.3 \pm 155.4 to $543.6 \pm 40.2)$ were observed after electrical stimulation of CEL ( $p<0.01$, Fig. 2 B and C).

\section{Effects of electrical stimulation of CEM and CEL} on neuronal spikes in DMNV

In response to electrical stimulation of CEM $(n=9)$, the FR of DMNV was significantly increased 


\section{A (CeM)}
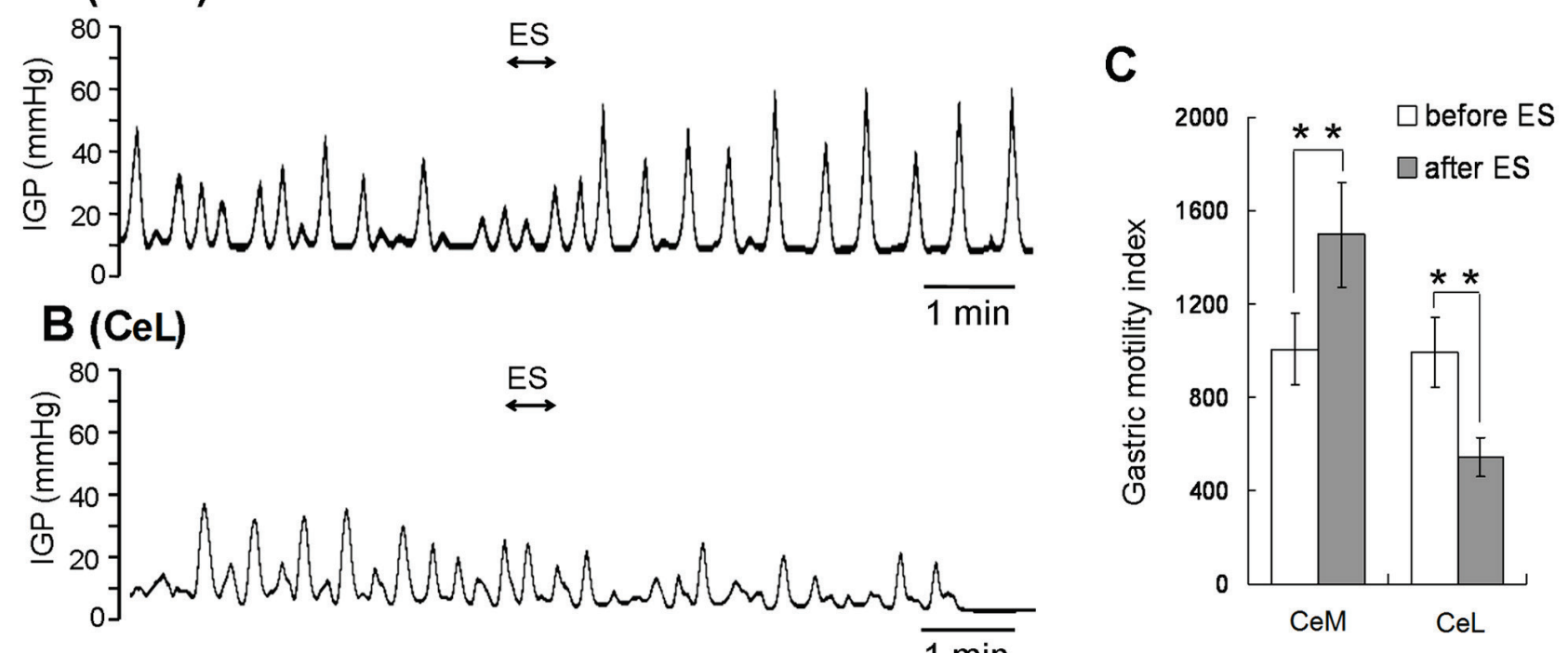

Fig. 2. Effects of electrical stimulation of CEM and CEL on gastric motility. Gastric motility curve of a rat recorded during the electrical stimulation of the CEM (A) and CEL (B); Gastric motility index (GMI) before and after stimulation of CEM ( $n=10)$ and CEL ( $n=10)$ groups, respectively $(\mathbf{C})$. Data represent the means $\pm \mathrm{SE} . * * p<0.01$. ES, electrical stimulation. IGP, intragastric pressure.

$(p<0.01 ; 2.77 \pm 0.30$ to $3.52 \pm 0.22$ spikes/s) (Fig. $3 \mathrm{~A}$ and $\mathrm{C}$ ). However, there was a significant decrease ( $p<0.05$; from $2.64 \pm 0.37$ to $1.78 \pm 0.24$ spikes/s) in the FR of DMNV after electrical stimulation of CEL $(n=9)$ (Fig. $3 \mathrm{~B}$ and $\mathrm{C}$ ).

Effects of electrical stimulation of CEM and CEL on neuronal spikes in $\mathrm{mNST}$

The FR of mNST was significantly decreased from $2.94 \pm 0.31$ to $2.01 \pm 0.38$ spikes/s $(p<0.01)$ in response to electrical stimulation of CEM $(n=8)$ (Fig. 4 A and C). By contrast, electrical stimulation of CEL $(n=9)$ caused the significant increase of FR in mNST from $3.02 \pm 0.31$ to $3.83 \pm 0.28$ spikes/s $(p<0.01)$ (Fig. 4 B and C).

\section{Discussion}

Considerable evidence has indicated that CNA is able to regulate gastric motility by modulating the neuronal activity in dorsal vagal complex (Zádori and Gyires 2013). It has been reported that stimulation of different regions of CNA can increase or inhibit gastric motility activity (Zádori and Gyires 2013). However, the role of amygdala in the regulation of gastrointestinal motor function is an understudied area. In the present study, electrical stimulation of CEM led to significant increase in IGP and increase in GMI, indicating a significant increase in gastric motility, while stimulation of CEL reduced gastric motility; furthermore, stimulation of either CEM or CEL also produced opposite influences on the neuronal activity in the DMNV and mNST of DVC.

Our finding here implies that stimulation of CEM can significantly increase gastric motility, and stimulation of CEL can significantly decrease gastric motility. The differences between the effects of stimulation of CEM and CEL on gastric motility might be attributed to the uneven distribution of CNA neurons projecting to the DVC. The difference has also been confirmed by what was previously reported by Lyubashina (2004) despite of the opposite observations. Lyubashina (2004) has reported that stimulation of CEM induced a predominant inhibitory effect on intragastric pressure in $59 \%$ of cases, with increases merely seen in $17 \%$ of cases, while stimulation of CEL caused decreases in intragastric pressure in $46 \%$ of cases and increases in $30 \%$ of cases, thus they proposed that stimulation of CNA can induce remarkable, differential alterations in intragastric pressure, with a predominantly inhibitory effect on performance of gastric reflex of interest. Furthermore, they have also observed that latent periods of the reactions after stimulation of CEM were $10.3 \pm 1.4$ (59\% cases) and $11.3 \pm 1.9 \mathrm{~s}$ (17\% cases) respectively, while latent periods of the reactions in response to CEL stimulation were $10.4 \pm 3.2$ (46\% cases) and $26.2 \pm 8.4 \mathrm{~s}$ (30\% cases). By contrast, the latent period of the reactions in response to either CEM or CEL 


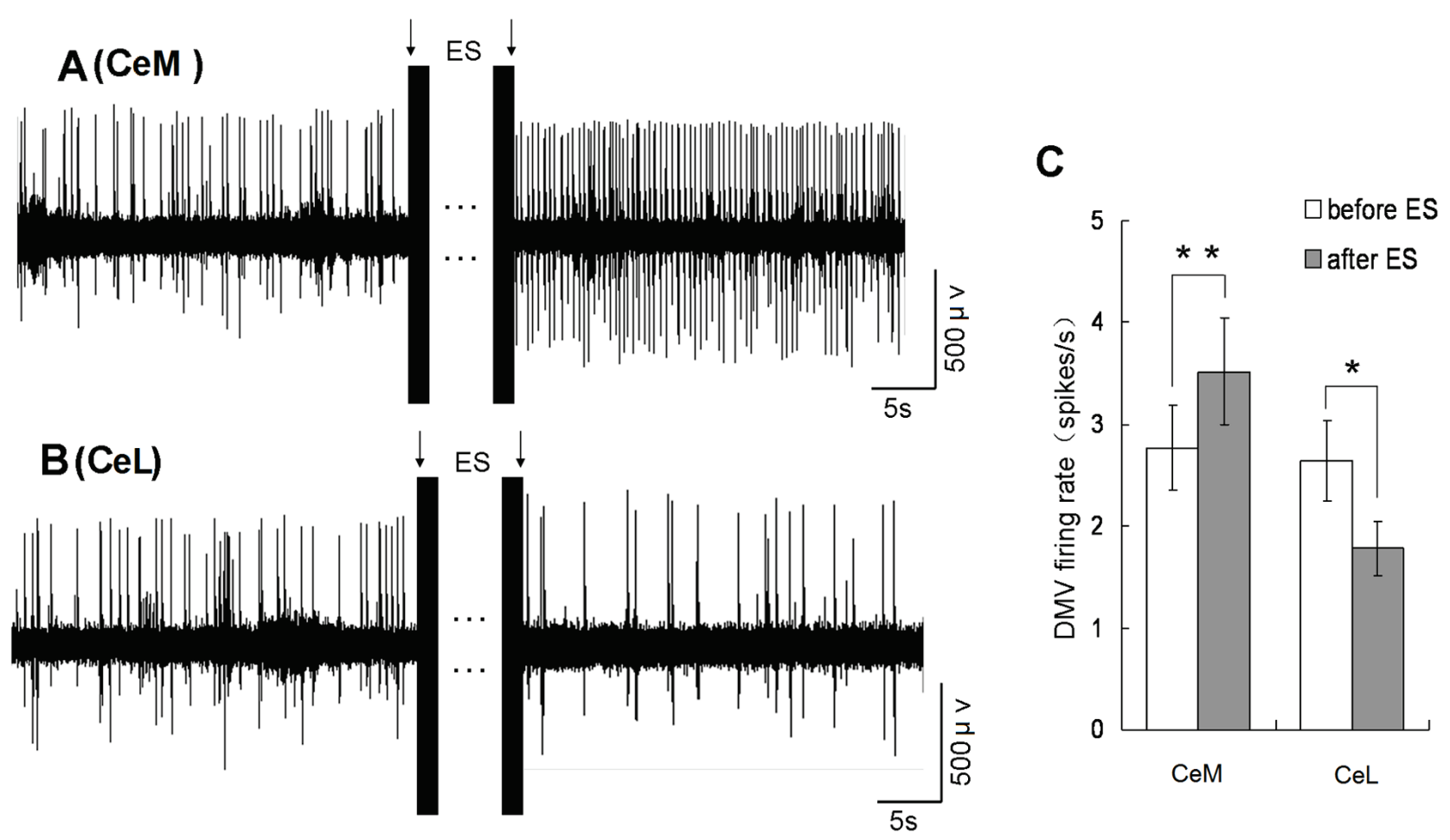

Fig. 3. Effects of electrical stimulation of CEM and CEL on neuronal spikes in DMNV. The original firing recording in the DMNV at 3 min before and after electrical stimulation of the CEM (A) and CEL (B); Firing rate (FR) at 3 min before and after stimulation in CEM ( $n=9$ ) and CEL $(n=9)$ groups, respectively $(\mathbf{C})$. Data represent the means \pm SE. $* p<0.05$ and $* * p<0.01$. ES, electrical stimulation.

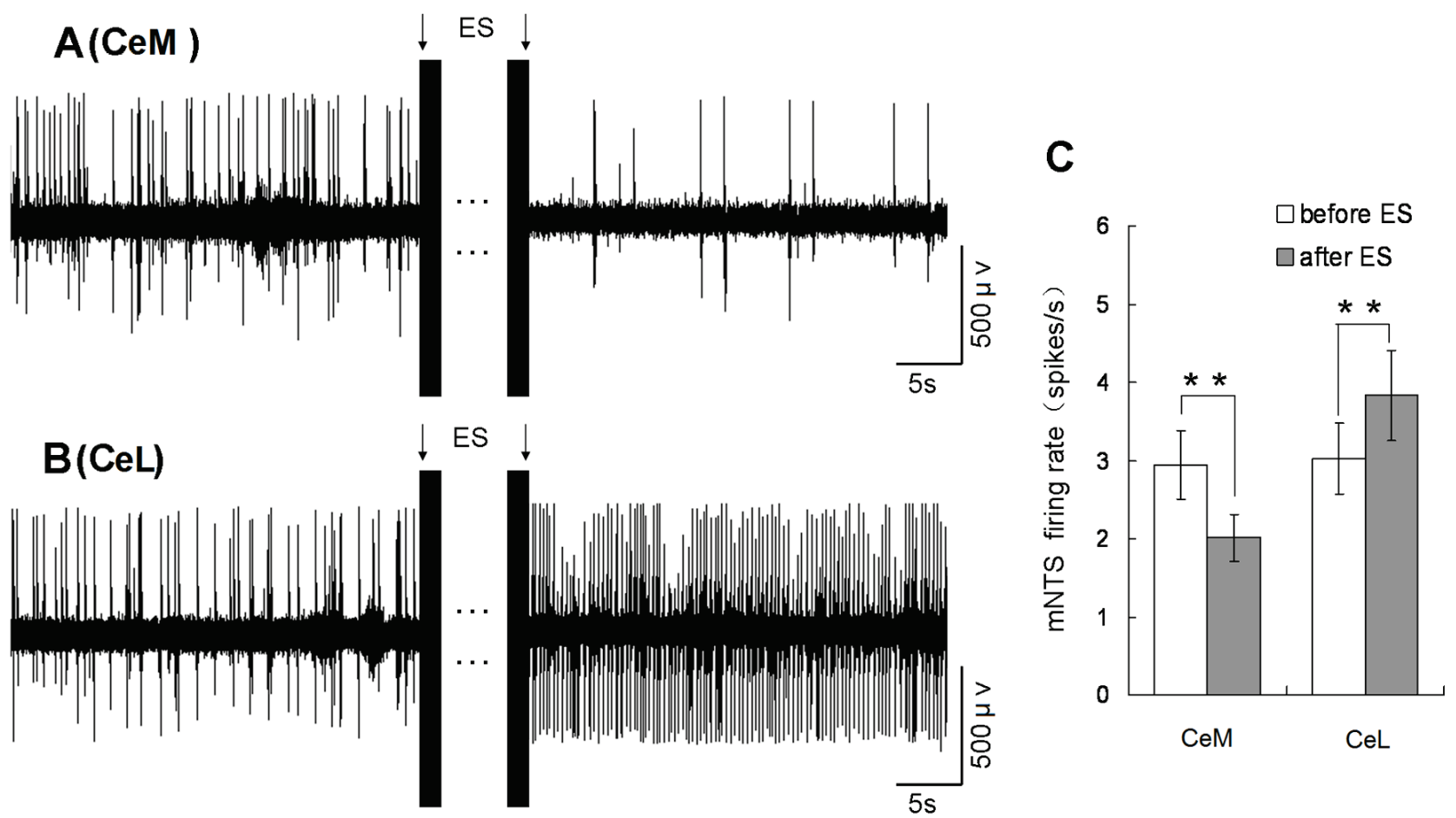

Fig. 4. Effects of electrical stimulation of CEM and CEL on neuronal spikes in $\mathrm{mNST}$. The original firing recording in the $\mathrm{mNST}$ at $3 \mathrm{~min}$ before and after electrical stimulation of the CEM (A) and CEL (B); Firing rate (FR) at 3 min before and after stimulation in CEM ( $n=8$ ) and CEL $(n=9)$ groups, respectively $(\mathbf{C})$. Data represent the means \pm SE. $* * p<0.01$. ES, electrical stimulation. 
stimulation observed here was approx. $3 \mathrm{~min}$. This difference may be due to the difference in the parameters of electrical stimulus. Additionally, we measured the intragastric pressure with rubber balloons, differing from the semiconductor pressure probes used by Lyubashina (2004) for measurement of intragastric pressure. Although balloons could monitor the changes in gastric motility as a whole, they can alter the intragastric pressure themselves inevitably and may also cause vagal excitement. The balloon method was still used for many reports, in which it was comprised of a control experiment (Zádori and Gyires 2013). By contrast, semiconductor pressure probes can avoid the above limitation, however, the position of the pressure probe could have a significantly effect on the measurement results of intragastric pressure. Lastly, differences in the physiological and emotional states may also induce the changes of gastric motor activity (Zhang et al. 2003). The body weight of Wistar rats were the same as in both studies. However, the body temperature of rats might be different during the whole experiments. Taken together, further work is needed to explore the factors that might be responsible for the reversal of effects seen in the present study compared to that of Lyubashina (2004).

In the present study, electrical stimulation of CEM significantly increased FR of DMNV, but significantly decreased FR of mNST; completely opposite results were observed in DMNV and mNST after electrical stimulation of CEL area. This implies electrical stimulation of the same CAN region has differing influences on CEM or DNMV neuronal activity. Electrophysiological and anatomic studies have revealed that efferent fibers from CNA terminate in both NST and DMNV, in regions that are involved in the regulation of gastrointestinal activity (Zhang et al. 2003), indicating that NST and DMNV might be engaged in the regulation of gastrointestinal activity via gastric vago-vagal reflex. Hermann et al. have revealed that mNST neurons are involved in the vago-vagal reflex and activation of mNST neurons can induce a dramatic decline in gastric motility activity (Hermann et al. 2005). The ipsilateral mNST and the subpostremal subnuclei of the NST are the primary targets of CNA axons, which are also the targets for the primary vagal afferent fibers from the gastrointestinal tract (Zhang et al. 2003, 2000). DMNV is considered to be the main source of descending projections from amygdala (Lyubashina 2004), and to the origin site of vagal efferent neurons that connect with upper gastrointestinal tract (Hornby and Wade 2011, Travagli et al. 2006). Zhang et al. (2003) and Liubashina et al. (2003) have reported that electrical stimulation of CNA inhibits NST neurons in rats, whereas Cox et al. (1986) have observed that stimulation of CNA can markedly excite NST neurons, indicating that stimulation of different CNA regions may have varied effects on DVC neurons. This hypothesis was confirmed by our finding that FR was markedly decreased in mNST, but was increased in DMNV in response to the electrical stimulation of CEM, which was opposite to what was observed after CEL stimulation. It has been reported that inhibitory response of DMNV neurons may be mediated by NST neurons, and inhibition of NST neurons by CNA stimulation may result in an increase in DMNV neuron activity (Babic et al. 2011). Thus, it may be further implied that amygdala may modulate DMV activity directly via projections or indirectly via mNST-mediated projections. Therefore, the neuronal spike responses of mNST and DMNV were always opposite under the electrical stimulation of either CEM or CEL in this study. What's more, anatomical and electrophysiological data demonstrate that inhibitory connections between NST and DMNV may play an important role in the regulation of gastrointestinal functions (Zhang et al. 2003). In addition, it has been indicated that CEM neurons are subjected to tonic inhibitory inputs, and that arises in CEL (Ciocchi et al. 2010, Pare and Duvarci 2012), supporting that effect of CEM and CEL stimulation on both gastric motility and neuronal spikes in DVC were also always opposite in the present study. Consequently, further investigation to clarify the underlying mechanisms of DVC modulating gastrointestinal functions is still needed. Microinjection of glutamate agonists into CNA subnuclei may be used in our future work to further confirm our observations.

In summary, electrical stimulation of CEM evoked gastric motility and caused the reduced neuronal spikes of mNST as well as increased neuronal spikes of DMNV, while CEL stimulation aroused completely contrary responses. The subdivisions of the CNA might play different roles in modulating neuronal spikes of DVC and in regulating gastric motility.

\section{Conflict of Interest}

There is no conflict of interest.

\section{Acknowledgements}

Research work was supported by the National Science Foundation of China (No. 31071920) and the Science Foundation of Shandong Province, China (No. ZR2011CM029). 


\section{References}

AWAN KL, RUTHERFORD JG: Gastric projecting neurons of dorsal (motor) nucleus of the vagus nerve (DMV) and their arborization. J Postgrad Med Inst (Peshawar-Pakistan) 18: 93-101, 2011.

BABIC T, BROWNING KN, TRAVAGLI RA: Differential organization of excitatory and inhibitory synapses within the rat dorsal vagal complex. Am J Physiol Gastrointest Liver Physiol 300: G21-G32, 2011.

BADRINARAYAN A, PRATER KE, ORSINI CA: The role of the central amygdala in selecting circuits and responses. J Neurosci 32: 8431-8433, 2012.

CIOCCHI S, HERRY C, GRENIER F, WOLFF SB, LETZKUS JJ, VLACHOS I, EHRLICH I, SPRENGEL R, DEISSEROTH K, STADLER MB: Encoding of conditioned fear in central amygdala inhibitory circuits. Nature 468: 277-282, 2010.

COX G, JORDAN D, MORUZZI P, SCHWABER J, SPYER K, TURNER S: Amygdaloid influences on brain-stem neurones in the rabbit. $J$ Physiol 381: 135-148, 1986.

CULLEN JJ, KELLY KA: Gastric motor physiology and pathophysiology. Surg Clin North Am 73: 1145-1160, 1993.

DUVARCI S, POPA D, PAR D: Central amygdala activity during fear conditioning. J Neurosci 31: 289-294, 2011.

GRANDI D, SOLENGHI E, GUERRINI R, POLIDORI C, MASSI M, MORINI G: Nociceptin/orphanin FQ prevents gastric damage induced by cold-restraint stress in the rat by acting in the periphery. Peptides 28: 1572-1579, 2007.

GRÈZES J, VALABRÈGUE R, GHOLIPOUR B, CHEVALLIER C: A direct amygdala-motor pathway for emotional displays to influence action: a diffusion tensor imaging study. Hum Brain Mapp 35: 5974-5983, 2014.

HAUBENSAK W, KUNWAR PS, CAI H, CIOCCHI S, WALL NR, PONNUSAMY R, BIAG J, DONG H-W, DEISSEROTH K, CALLAWAY EM: Genetic dissection of an amygdala microcircuit that gates conditioned fear. Nature 468: 270-276, 2010.

HERMANN GE, NASSE JS, ROGERS RC: $\alpha-1$ adrenergic input to solitary nucleus neurones: calcium oscillations, excitation and gastric reflex control. $J$ Physiol 562: 553-568, 2005.

HORNBY PJ, WADE PR: Central control of gastrointestinal function. In: Central Regulation of Autonomic Functions. LLEWELLYN-SMITH IJ, VERBERNE AJM (eds), Oxford University Press, Oxford, 2011, pp 259-273.

HUERTA-FRANCO MR, VARGAS-LUNA M, MONTES-FRAUSTO JB, MORALES-MATA I, RAMIREZPADILLA L: Effect of psychological stress on gastric motility assessed by electrical bio-impedance. World $J$ Gastroenterol 18: 5027-5033, 2012.

INSTITUTE FOR LABORATORY ANIMAL RESEARCH, NATIONAL RESEARCH COUNCIL: Guide for the Care and Use of Laboratory Animals. National Academy Press, Washington, D.C., 1996.

KIM HM, CHOI JS, CHO JH: A pilot trial of ambulatory monitoring of gastric motility using a modified magnetic capsule endoscope. J Neurogastroenterol Motil 20: 261-264, 2014.

KIM MJ, LOUCKS RA, PALMER AL, BROWN AC, SOLOMON KM, MARCHANTE AN, WHALEN PJ: The structural and functional connectivity of the amygdala: from normal emotion to pathological anxiety. Behav Brain Res 223: 403-410, 2011.

LIUBASHINA O, JOLKKONEN E, PITKÄNEN A: Projections from the central nucleus of the amygdala to the gastric related area of the dorsal vagal complex: a Phaseolus vulgaris-leucoagglutinin study in rat. Neurosci Lett 291: $85-88,2000$.

LIUBASHINA O, BAGAEV V, KHOTIANTSEV S: Amygdalofugal modulation of the vago-vagal gastric motor reflex in rat. Neurosci Lett 325: 183-186, 2002.

LYUBASHINA OA: Possible mechanisms of involvement of the amygdaloid complex in the control of gastric motor function. Neurosci Behav Physiol 34: 379-388, 2004.

PARE D, DUVARCI S: Amygdala microcircuits mediating fear expression and extinction. Curr Opin Neurobiol 22: 717-723, 2012.

PAXINOS G, WATSON C: The Rat Brain in Stereotaxic Coordinates. Academic Press Limited, London, 1998.

PORCELLI P, DE CARNE M, LEANDRO G: Alexithymia and gastrointestinal-specific anxiety in moderate to severe irritable bowel syndrome. Compr Psychiatry 55: 1647-1653, 2014. 
PURGERT RJ, WHEELER DS, MCDANNALD MA, HOLLAND PC: Role of amygdala central nucleus in aversive learning produced by shock or by unexpected omission of food. J Neurosci 32: 2461-2472, 2012.

RINAMAN L, KOEHNLE TJ: Development of central visceral circuits. In: Oxford Handbook of Developmental Behavioral Neuroscience. BLUMBERG MS, FREEMAN JH, ROBINSON SR (eds), Oxford University Press, New York, 2010, pp 298-321.

STANGHELLINI V, TOSETTI C, BARBARA G, DE GIORGIO R, COGLIANDRO L, COGLIANDRO R, CORINALDESI R: Dyspeptic symptoms and gastric emptying in the irritable bowel syndrome. Am J Gastroenterol 97: 2738-2743, 2002.

TALLEY NJ, LOCKE GR, LAHR B, ZINSMEISTER AR, TOUGAS G, LIGOZIO G, ROJAVIN MA, TACK J: Functional dyspepsia, delayed gastric emptying, and impaired quality of life. Gut 55: 933-939, 2006.

TRAVAGLI RA, HERMANN GE, BROWNING KN, ROGERS RC: Brainstem circuits regulating gastric function. Annu Rev Physiol 68: 279-305, 2006.

TYE KM, PRAKASH R, KIM S-Y, FENNO LE, GROSENICK L, ZARABI H, THOMPSON KR, GRADINARU V, RAMAKRISHNAN C, DEISSEROTH K: Amygdala circuitry mediating reversible and bidirectional control of anxiety. Nature 471: 358-362, 2011.

VENTURA-SILVA AP, MELO A, FERREIRA AC, CARVALHO MM, CAMPOS FL, SOUSA N, PEGO JM: Excitotoxic lesions in the central nucleus of the amygdala attenuate stress-induced anxiety behavior. Front Behav Neurosci 7: 32, 2013. DOI: 10.3389/fnbeh.2013.00032

ZÁDORI ZS, GYIRES K: In vivo measurement of intragastric pressure with a rubber balloon in the anesthetized rat. Curr Protoc Toxicol 57: Unit 21.12., 2013. DOI: 10.1002/0471140856.tx2112s57

ZHANG X, RENEHAN WE, FOGEL R: Vagal innervation of the rat duodenum. J Auton Nerv Syst 79: 8-18, 2000.

ZHANG X, CUI J, TAN Z, JIANG C, FOGEL R: The central nucleus of the amygdala modulates gut-related neurons in the dorsal vagal complex in rats. $J$ Physiol 553: 1005-1018, 2003. 